\title{
The Mass-Ratio Distribution of Visual Binary Stars
}

Published in: Astrophys. Space Sci. 173: 315-342, 1990

\begin{abstract}
The selection effects that govern the observations of Visual Binary Stars are investigated, in order to obtain a realistic statistical distribution of the mass-ratio $q=M_{\mathrm{sec}} / M_{\text {prim }}$. To this end a numerical simulation programme has been developed, which 'generates' binary stars and 'looks' at them to determine whether an observer on Earth would be able to detect them. The simulations show that for mass-ratios $q>0.35$, observations are expected to reveal the real $q$-distribution, while for massratios $q<0.35$ selection effects begin to play a major part. It is found that the observed mass-ratio distribution for main-sequence systems, derived from the Index Catalogue of Visual Binary Stars (IDS), can be explained by a distribution of secondary masses according to the Initial Mass Function (IMF), i.e. $\phi(M) \propto M^{-2.7}$.

From the Fourth Catalogue of Orbits of Visual Binary Stars (OVB) authors find a $q$-distribution that peaks strongly for $q$-values close to $q=1$. It is shown that this mass-ratio distribution may be the result of a sampling selection effect. Due to this sampling selection effect, the OVB is a considerably more biased sample of the binary population in our Galaxy than the IDS. Numerical simulations of biased sampling show that the $q$-distribution, found from the OVB, is not incompatible with the distribution of secondary masses according to the IMF (for $q>0.35$ ), found from the IDS.

Because of the selection effects, it is difficult to establish the real $q$-distribution for $q<0.35$. If the real $q$-distribution departs from $\phi(q) \propto q^{-2.7}$ for $q \leq 0.35$, about $36 \%$ of all stars are in visual binaries (i.e. if the $q$-distribution is assumed to be flat for $0<q<0.35$ ); if the distribution flattens for $q \leq 0.25$, about $60 \%$ of the stars must be primaries of visual binaries.
\end{abstract}




\subsection{Introduction}

In the past two decades, a variety of scenarios for the evolution of close binary stars have been developed. It is hard to determine how often the processes described by these scenarios will occur in our galaxy, or in parts of it: in associations, open clusters or globular clusters. One way to determine the validity of an evolutionary scenario, is to look at the statistical properties of double stars. An evolutionary scenario for a well defined system often makes predictions about the end product, intermediate stages and the life times of such stages. When we compare the occurrence of possible predecessors, candidate intermediate systems and assumed end products, the likelihood of a scenario can be established.

To make an assessment as described above, one needs a sound base of statistical data on binary star properties. A first inventory of such data has been made by Kuiper (1935a, 1935b). He tried to determine to what extent the data on Visual Binaries and Spectroscopic Binaries were complete at the time. In spite of a sometimes dramatic increase in the amount of data available, most of Kuiper's assessments of the completeness of the data still hold.

In this paper we concentrate on the distributions of parameters of visual binaries; a subsequent paper will deal with the mass-ratio distribution of spectroscopic binaries. Modern statistical data for parameters of visual binary stars have been presented by Vereshchagin et al. (1987, 1988), Poveda et al. (1982), Trimble and Walker (1986) and Kraicheva et al. (1985).

Vereshchagin et al. and Poveda et al. derived their data from the Index Catalogue of Visual Double Stars (IDS) by Jeffers, Van den Bos and Greeby (1963), a predecessor of the The Washington Double Star Catalogue (WDS) by Worley and Douglass ${ }^{1}$ (1984). Vereshchagin et al. derived their data from a filtered version of the IDS. This filtered IDS contains luminosity class $\mathrm{V}$ systems only, and optical pairs have been discarded from the catalogue, reducing the probability for any given pair of stars in the catalogue to be an optical pair to less than $1 \%$.

Trimble and Walker (1986) derive their statistics from the systems with known spectral types in the Fourth Catalogue of Orbits of Visual Binary Stars (OvB) by Worley and Heintz (1983).

Important parameters with respect to binary star evolution are the semi-major axis of the orbit $a$ and the ratio of the masses of the components: $q=M_{\mathrm{sec}} / M_{\text {prim }}$. For the mass-ratios, statistics from the OVB and the IDS yield totally different distributions. Vereshchagin et al. (1987) find a mass-ratio-distribution from the IDS, which for $q>0.35$ is in agreement with a distribution of secondary masses according to the Initial Mass Function (Scalo 1986), i.e. $d N / d q \propto q^{-2.7}$. Figure 1.1.a shows the results obtained by Vereshchagin et al. for different (magnitude limited) sub-samples of the IDS. Trimble and Walker (1986) find from the OVB a $q$-distribution with a pronounced peak near $q=1$, as shown in Fig. 1.1.b.

It is well known that observations of visual binary stars are subject to severe

\footnotetext{
${ }^{1}$ In 1976 the U.S. Naval Observatory issued a version of the IDS on magnetic tape, called IDS II. This catalogue was compiled by Worley. In 1984 the U.S. Naval Observatory issued the Washington Double Star Catalogue, compiled by Worley and Douglass. The IDS has since then been incorporated into the WDS.
} 


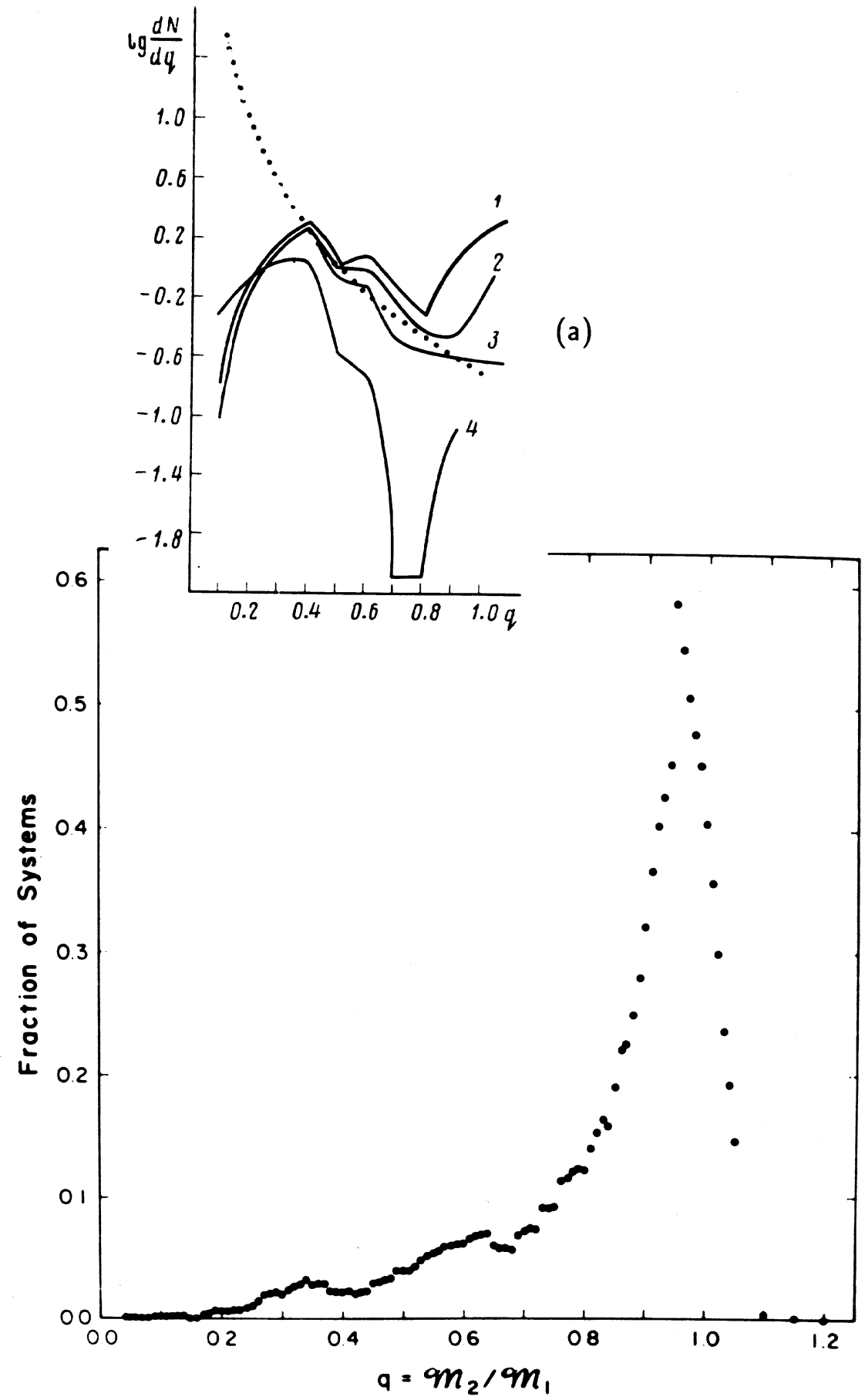

(b)

Figure 1.1: Observed $q$-distributions, not corrected for selection effects, as derived from catalogues. (a) From the IDS, for luminosity class V (main-sequence) systems, by Vereshchagin et al. (1987). The four curves are for: 1. all systems in the filtered IDS. 2. systems with primary magnitudes $m_{\text {prim }}<9^{m} ; 3 . m_{\text {prim }}<7^{m}$; and 4. $m_{\text {prim }}<5^{m}$. The dotted line represents the IMF. (b) From the OVB by Trimble and Walker (1986). 
selection effects. Because of this, the distributions of binary star parameters as derived from observations, may be very different from the real distributions. If the selection effects were known, it would be possible to assess the real distributions of binary star parameters. In this paper, an assessment of the selection effects is made, in an attempt to resolve the apparent contradiction of the $q$-distributions as obtained from the WDS and the OVB. To this end, a numerical programme has been developed that 'generates' double star systems and then 'looks' at them to determine whether an observer on Earth would be able to detect them. This paper describes how binary star parameters were generated (section 1.2), the selection effects that were taken into account (section 1.3), the results of the simulations (sections 1.4 and 1.5), and finally it discusses the consequences the simulations may have for our understanding of the real distributions of binary star parameters (section 1.6).

Details of the programme are given in the appendix. Copies of the programme, which was written in FORTRAN, are available upon request.

\subsection{Binary star parameters}

The orbit of a binary system is fully determined by seven parameters. These are: masses of primary and secondary components $M_{1}$ and $M_{2}$, semi-major axis of the relative orbit $a$, eccentricity $e$, inclination of the orbit $i$, longitude of periastron $\omega$, and epoch of periastron passage $T$. An important parameter regarding selection effects is the distance of the binary system to the observer. We introduce this distance $d$ as an eighth parameter.

From the basic parameters other parameters of interest can be derived, such as the period $P$ and mass-ratio $q=M_{2} / M_{1}$. With respect to binary star evolution, the semi-major axis of the orbit $a$ and the mass-ratio $q$ are of special interest. They determine whether Roche lobe overflow will occur, and the rate of mass exchange. Because of their importance, there will be an emphasis on these two parameters in this paper.

\subsubsection{Visual binary parameters}

For visual binary stars there are two distinct sets of data. The OvB lists binary stars for which the orbital elements have been determined, the WDS contains data of stars that are likely to be binaries on the basis of statistical arguments, but for which in most cases orbital motion has not been observed.

I will call the systems in the WDS statistical doubles. There are data for 73610 double stars in the 1984 version of the WDS. In contrast, there are 928 computed orbits for 847 binary systems in the OVB.

When the semi-major axes $a_{1}$ and $a_{2}$ of the orbits of both components around the center of mass are known separately, the mass-ratio can be calculated from:

$$
q=\frac{M_{2}}{M_{1}}=\frac{a_{1}}{a_{2}}
$$

Unfortunately, there are only very few visual binary systems for which these elements are known. The OVB only contains information about the semi-major axis $a=a_{1}+a_{2}$ 
of the relative orbit of the components around each other. For the majority of visual binaries, both from the OVB and the WDS, authors have to resort to a mass-luminosity relation (Trimble and Walker 1986), or a spectral-type-mass relation (Vereshchagin et al. 1988) to determine masses and mass-ratios.

The major selection effect for visual binaries is the magnitude difference of the components with respect to their angular separation. When the angular separation of the components is less than the resolution of the telescope, the system cannot be recognised as double. When at a given angular separation the magnitude difference is too large, the secondary can no longer be detected in the presence of its 'bright' primary. In order to determine the completeness of either OVB or WDS, it is important to assess this relation between magnitude difference and minimum separation.

\subsection{Simulating selection effects}

In order to assess the observational selection effects, a computer programme has been developed, in which the most important selection effects were incorporated. The programme 'generates' double stars and then 'looks' at them to determine whether they can be detected as visual binaries. An outline of the programme is given below.

This study is restricted to main-sequence stars, in order to avoid confusion between evolutionary and selection effects. Therefore only physics for main-sequence stars has been incorporated in the programme. First observations of statistical doubles are simulated in Sect. 1.4. The selection effects for visual binaries with computed orbits will be addressed in Sect. 1.5.

\subsubsection{Outline}

Binary stars were 'generated' by producing the eight basic parameters mentioned in section 1.2 from random variates. I will call the basic parameters 'generated parameters'. In Sect. 1.3.2 an account of the distributions that have been used is given. The process by which a given distribution is reproduced by a random variate is described in the appendix, section 1.A.2. The appendix also contains the mathematics involved in turning specific distributions of binary star parameters into random variates.

From the generated parameters, 13 other parameters were calculated, i.e. period of the system $P$, apparent magnitude of primary and secondary $m_{1}$ and $m_{2}$, difference in apparent magnitude $\Delta m$, spectral types of the components Sp1 and Sp2, mass ratio $q=M_{2} / M_{1}$, radii $R_{1}$ and $R_{2}$, luminosities $L_{1}$ and $L_{2}$, and effective temperatures $T_{1}$ and $T_{2}$,

I will call these 13 parameters 'derived parameters'. Not all 13 derived parameters were used in the simulations of the selection effects for visual binaries. However, the programme was set up to investigate the selection effects for three observational classes of binaries: spectroscopic, visual and eclipsing. The selection effects of spectroscopic and eclipsing binaries will be the subjects of separate papers. How derived parameters are calculated from generated parameters, is explained in the appendix, section 1.A.5. 
The crucial parameter with respect to observations of visual binaries, is the angular separation $\rho$. This is a fourteenth parameter, which can be calculated from previously mentioned parameters.

The programme 'looks' at three parameters to check whether the binary can be detected as a visual binary by an observer on earth. These are primary magnitude $m_{\text {prim }}$, angular separation $\rho$, and magnitude difference $\Delta m$. The criteria by which it does this are discussed in section 1.3.3.

\subsubsection{Distributions of Generated Parameters}

In general, distributions derived from catalogues are presented as histograms, displaying $N(x)$ in intervals $\Delta x$ for parameter $x$. It is useful to define a probability density function (PDF) $d N(x) / d x=\phi(x)$. The number of elements in a finite interval $x, x+\Delta x$ can be expressed as an integral of the PDF:

$$
N_{x, x+\Delta x}=\int_{x}^{x+\Delta x} \phi\left(x^{\prime}\right) d x^{\prime}
$$

Throughout this paper, distributions will be defined by their probability density functions. Real (= input) distributions will be denoted by $\Phi(x)$; distributions derived from observations (catalogues) will be denoted by $\phi(x)$. Distributions that result as 'observed' distributions from computer simulations will be denoted by $\phi_{s}(x)$.

For the 'generated' parameters the following distributions were adopted:

Primary mass. Primary masses are assumed to be distributed according to the Initial Mass Function. Kraicheva et al. (1985) and Popova et al. (1982) produce observational evidence for such a distribution. I have adopted the initial mass function proposed by Scalo (1986). Primary masses $M_{1}$ were generated in the range $1 M_{\odot}<M_{1}<30 M_{\odot}$.

Secondary mass. The secondary mass distribution is imposed by the mass-ratio distribution.

Mass-ratio. The PDF of the mass-ratio distribution, $\Phi(q)$ is represented by a power law, i.e., $\Phi(q)$ is proportional to $q^{-\alpha}$. Results for three different values of $\alpha$ are presented in this paper:

- $\alpha=2.7$ : This means that the secondary masses are also distributed according to the IMF. For $q \rightarrow 0, q^{-2.7}$ tends to infinity. Therefore, a lower limit $q_{0}$ had to be set to the generated $q$-values. I have taken $q_{0}=0.05$. Systems with $q<0.05$ are hardly ever detected, because of the selection effects that affect the observed $q$-distribution for $q<0.35$.

- $\alpha=0$ : This value of $\alpha$ represents a flat $q$-distribution. It means that the mass of the secondary has an equal probability to have any value between zero and the mass of the primary $M_{\text {prim. }}$.

- $\alpha=-1.5$ : This value of $\alpha$ produces a distribution of secondary masses with a behaviour quite contrary to that of the IMF. The secondary masses will preferentially have values close to the mass of the primary. This distribution is suggested by Popov in a Ph.D. thesis (Popova et al. 1982). 
The 'observed' distributions with $\mathrm{PDF} \phi_{s}(q)$ that result from simulations with these three extreme 'input' distributions, can be compared with distributions obtained from real observations, and thus provide an indication of the real $q$-distribution $\Phi(q)$ of binary systems in our galaxy.

Semi-major axis. The semi-major axis $a$, of the relative orbit of binary systems, is assumed to be distributed proportional to $1 /$ a, according to Vereshchagin et al. (1987, 1988). Values of $a$ were generated in the range $1.4 R_{\text {Roche }}<a<10^{6} R_{\odot}$. Here $R_{\text {Roche }}$ is the radius of a sphere with a volume equal to the Roche volume (Pringle and Wade 1985). The lower limit makes sure that the generated binary components are not in contact. The value of $10^{6} R_{\odot}$ for $a_{\max }$ is adopted here to represent an upper limit: wider systems would be disrupted by tidal forces induced by the Galaxy. For a discussion of $a_{\max }$ see Wasserman (1988).

Eccentricity. The eccentricity of the orbits $e$ was taken equal to zero. This was done for reasons of simplicity, and to get a first order impression. The simulation programme does, however, allow for distributions of $e$ in the interval $(0,1)$.

Inclination. The inclination of the orbits of the binary systems was taken from a distribution that allows the normal vector, perpendicular to the orbital plane, to point in any direction. This choice was made because there is no clear evidence for any alignment of angular momentum vectors of binary systems within our galaxy.

Longitude of periastron and Epoch of periastron passage. Not of importance here as we adopted circular orbits.

Distance. The distance is taken to be distributed according to a homogeneous distribution of stars in space, in a volume with a radius stretching from $d=0$ to 50 parsec. The maximum value $d_{\max }=50 \mathrm{pc}$ was adopted because $\mathrm{F}, \mathrm{G}$ and $\mathrm{K}$ type stars have magnitudes $m>7^{m}$ when they are at greater distances (the value of $m=7^{m}$ is implemented as a selection effect (section 1.3.3), and most systems in the WDS and the OVB are of spectral types (A), F, G, and K). Since the galaxy has a thickness of about $400 \mathrm{pc}$ at the location of the sun, it was allowed to assume a spherical volume.

\subsubsection{Selection Effects}

\section{Maximum primary magnitude}

In the statistical analyses of visual binary catalogues, authors often put restrictions on the sample they use. One such restriction is a maximum magnitude for the systems considered. Vereshchagin et al. $(1987,1988)$ consider the sample of systems in the WDS with primary magnitudes $m_{\text {prim }}<7^{m}$ the most complete and unbiased one. Therefore, systems with primaries fainter than $7^{m}$ are also rejected in the selection effects simulation programme.

\section{Minimum and maximum separation}

When the binary nature of an optical pair is decided on statistical arguments, as it is done for stars in the WDS, it is well known that two selection effects are of major importance: 1 . the minimum separation at which two stars can be distinguished, and 


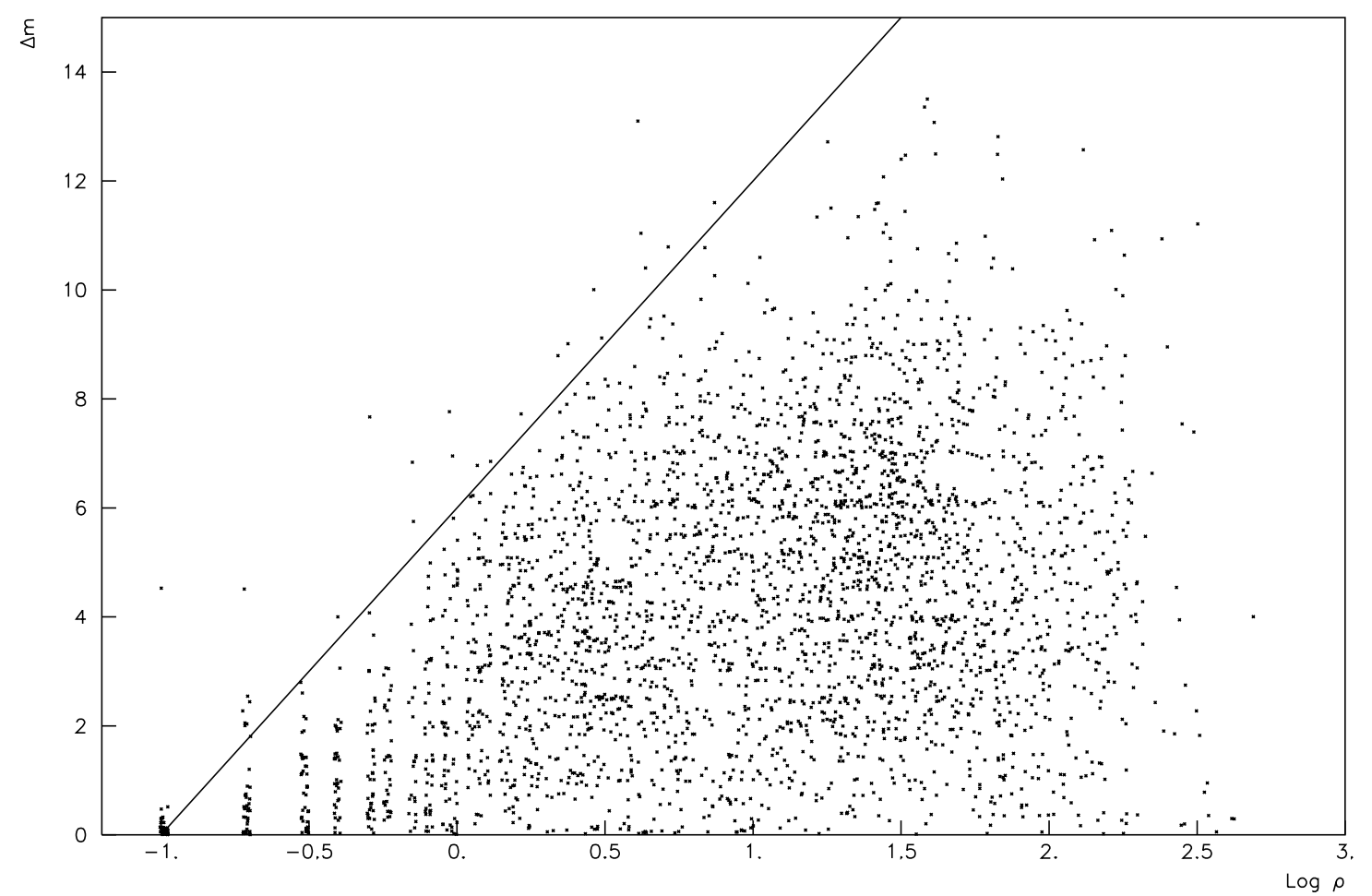

Figure 1.2: Scatter diagram of $\log \rho$ vs. $\Delta m$, for all systems in the WDS with primaries brighter than $7^{m}$. The solid line represents $\log \rho_{\min }=\Delta m / 6-1$.

2. the maximum separation at which there is no confusion with field stars, at least to a certain level of confidence. Both the minimum and the maximum separation depend on the difference in magnitude of the stars considered.

Most of the observations of visual binary stars in the WDS were made visually. The telescopes used had apertures in the range of 50 to 90 centimeters. The minimum detectable separation is, for any given magnitude difference of the components of the binary star, determined by the performance of the combination of telescope and observer. Öpik (1924) and Heintz (1969) have determined the minimum detectable separation from observations available at that time. However, what in fact is needed, is an independent assessment of the performance of the eye-telescope system, since we try to determine statistical properties of binary stars from these same observations. I return to this problem in the discussion, section 1.6.1.

Kuiper (1935a) presents a scatter diagram, in which the logarithm of the angular separation $\rho$ is plotted against the magnitude difference $\Delta m$, for a number of binary systems. A line can be drawn in this diagram, indicating a minimum separation $\rho_{\text {min }}$ at a given value for the magnitude difference. Figure 1.2 is a modern version of the $\log \rho-\Delta m$ diagram, which contains all systems in the WDS with primary magnitudes $m_{\text {prim }} \leq 7^{m}$. Also the line $\log \rho_{\text {min }}=\Delta m / 6-1$ has been drawn. As in the $\Delta m$ range $0^{m}$ to $10^{m}$ this line appears to form the clear boundary between the filled and the empty part of the diagram, I have adopted this line to represent the limit for the minimum detectable angular separation for visual binaries.

For the maximum angular separation $\rho_{\max }$ at which an observed pair can still be 

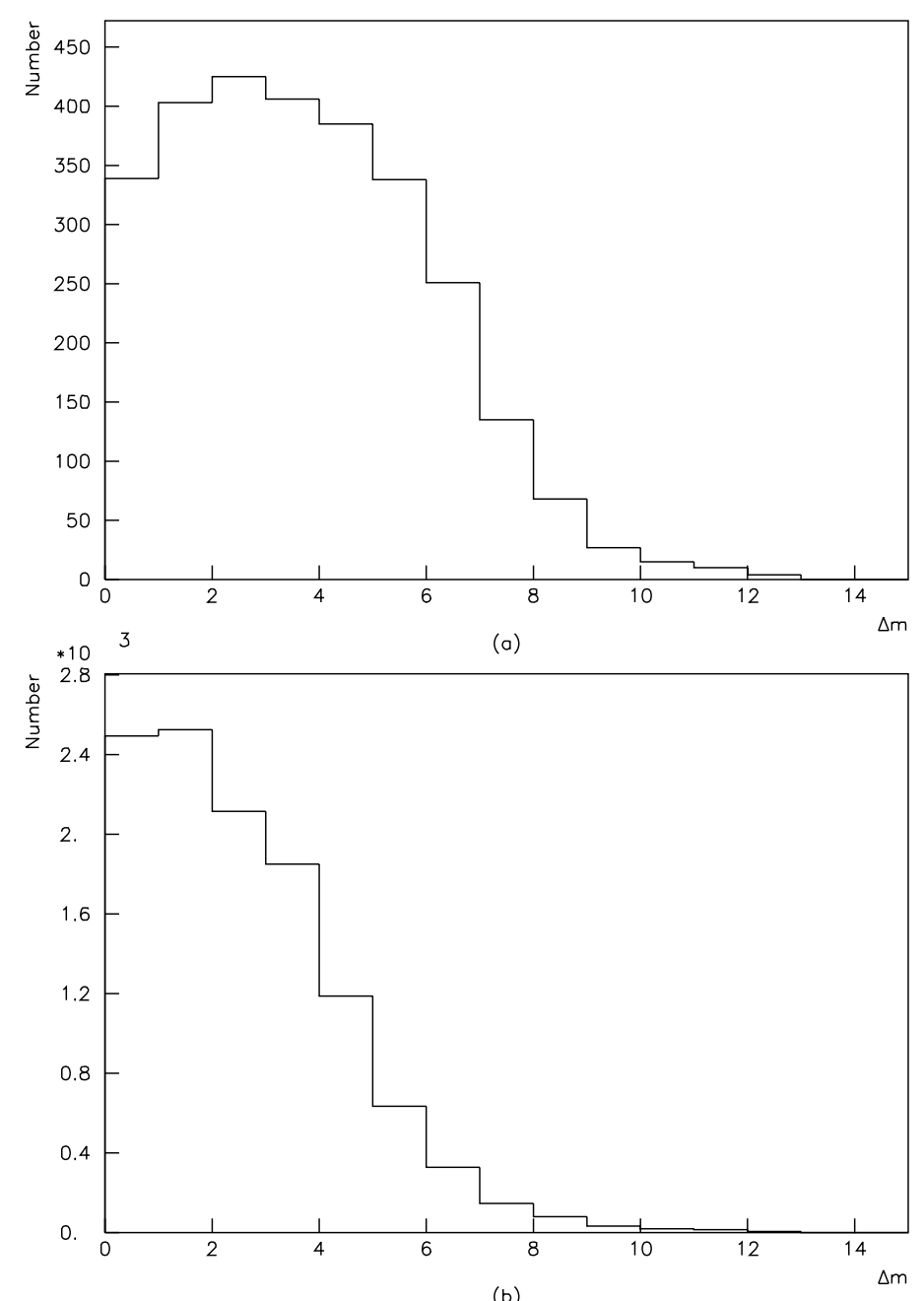

(b)

Figure 1.3: Histograms of $\Delta m$ for systems in the WDS. (a) For systems with primary magnitudes $m_{\text {prim }}$ up to $7^{m}$. (b) For systems with $m_{\text {prim }}$ up to $9^{m}$.

considered a binary system, several statistical confidence limits have been proposed. Aitken (1932) proposed a formula for $\rho_{\max }$ as a function of the combined magnitude $m$ of the components: $\log \rho_{\max }=2.8-0.2 m$. The Aitken criterion may be considered a modest one, stricter than some criteria, but less so than others (cf. Poveda et al. (1982)). It is for this reason that I have incorporated it as the selection effect for the maximum value of detectable angular separation.

\section{Maximum magnitude difference}

It turned out from the simulations (described in the subsequent sections) that the selection effects mentioned in the previous sections could only account for the general slope of the $q$-distribution for $q>0.35$. The turnover at $q=0.35$, that is seen in distributions derived from the WDS (Vereshchagin et al. 1987, Vereshchagin et al. 1988), could not be reproduced until a maximum magnitude difference was introduced. 
Figure 1.3.a shows the histogram of magnitude differences for systems in the WDS with primaries up to magnitude $7^{m}$. This histogram shows that there is a maximum magnitude difference of about $5^{m}$. The cut off is not sharp, but the number of systems drops rapidly for $\Delta m$ in the range from $6^{m}$ to $9^{m}$. This behaviour of $\Delta m$ was incorporated into the selection effects simulations by generating a maximum value $\Delta m_{\max }$ in the range $6^{m}$ to $9^{m}$ by means of a normal distribution (appendix, section 1.A.3). Of course, it is not obvious that a maximum in the histogram for $\Delta m$ should be due to selection effects. This problem will be addressed in the discussion.

\subsection{Statistical doubles}

To obtain their observed mass-ratio distribution, Vereshchagin et al. used a modified version of the IDS. It has been pointed out in section 1.2.1, that the IDS contains visual double stars, which are assumed to be physical binaries on statistical grounds. Some authors have argued that up to $30 \%$ of the systems in the IDS may not be physical doubles (Poveda et al. 1982, and references therein). Poveda et al. devised a filtering technique to discard optical pairs from the catalogue. They claim that the filtered version of the IDS contains less than $1 \%$ non-physical binary systems. It is a similar filtering technique that has been employed by Vereshchagin et al. for their statistical analysis of the physical parameters of visual binaries from the IDS. For the observed $q$-distribution (not corrected for selection effects) Vereshchagin et al. present four curves, involving different magnitude-limited sub-samples of the filtered IDS. For $q>0.35$ all curves show a slope which agrees with the IMF. Three of these four curves show a second maximum in the $q$-distribution toward $q=1$. The nature and significance of this second maximum will be addressed in the discussion, section 1.6.4.

\subsubsection{Assessment of the selection effects}

Vereshchagin et al. (1987) consider the distribution which they derived for the systems with $m_{\text {prim }} \leq 7^{m}$ the most complete and unbiased one. It is this distribution (nr. 3 in Fig. 1.1.a) which for $0.4<q \leq 1$ follows the IMF most closely. However, because they are not certain about the selection effects, Vereshchagin et al. indicate that the observed distribution might still be accounted for by a real mass-ratio distribution which is flat or even rising toward $q=1$.

It will be shown below, that by applying the four selection effects described in Sect. 1.3.3 - i.e. a maximum primary magnitude, a minimum and a maximum angular separation related to the magnitude difference of the components, and a maximum magnitude difference - Vereshchagin et al's distribution nr. 3 is reproduced well only for an input $q$-distribution similar in shape to the IMF.

\subsubsection{Results}

The results of the simulations for systems in the WDS, the statistical doubles, are presented in Figs. 1.4 to 1.6. The figures are subdivided into figures $a$ and $b$, representing: $a$. the generated distributions with $\operatorname{PDFs} \Phi(q)$ and $b$. the distributions from 


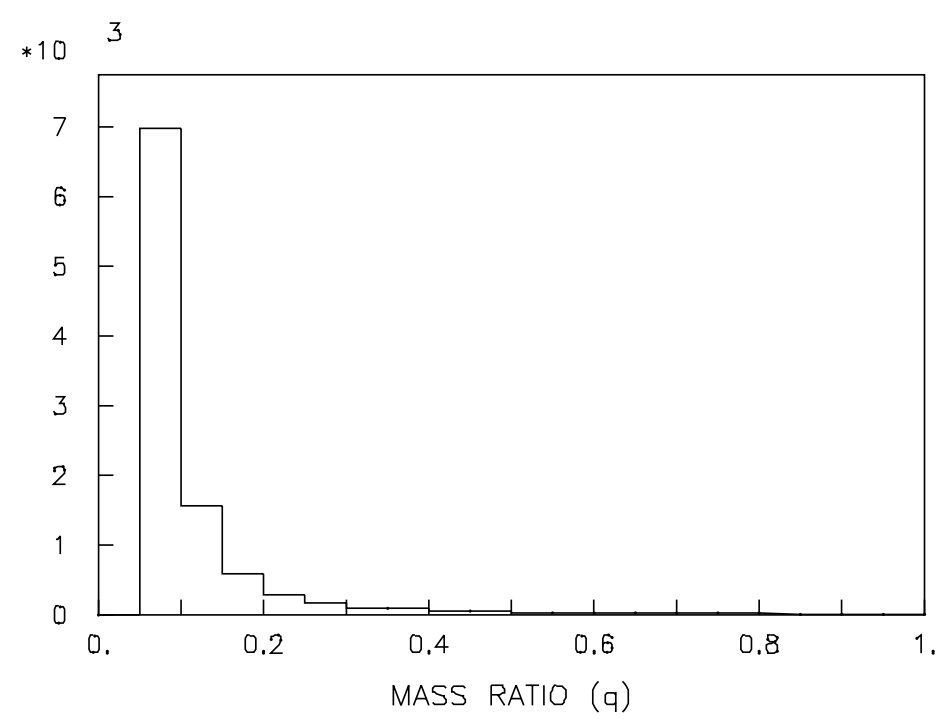

(a)

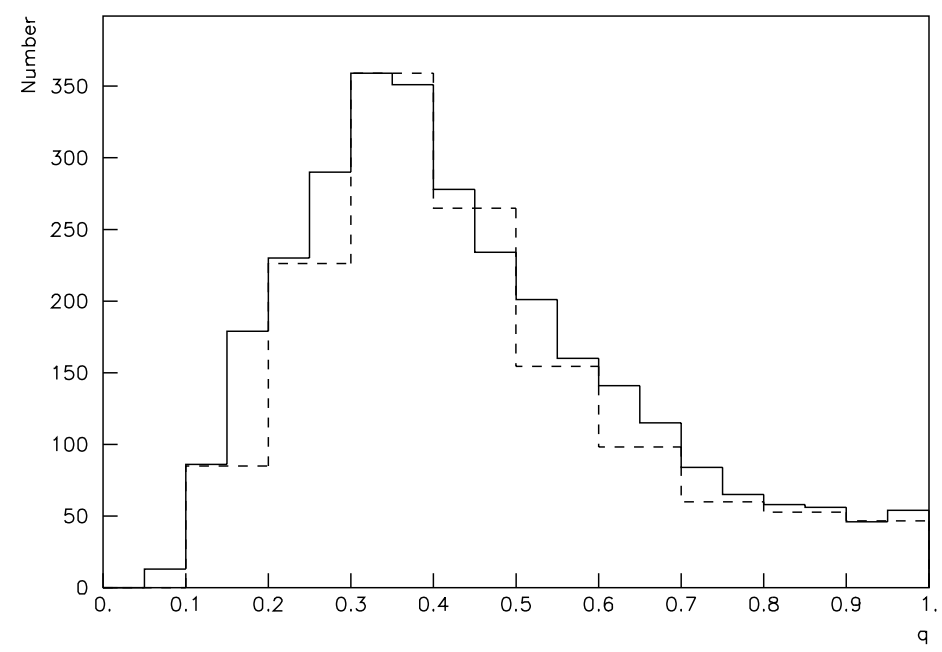

(b)

Figure 1.4: Simulations with PDF $\Phi(q)$ according to the IMF. (a) Generated $q$-distribution. (b) $q$-Distribution obtained for simulated observations. The dashed line represents observed distribution nr. 3 of Vereshchagin et al. (1987) from figure 1.1.a.

simulated observations, with PDFs $\phi_{s}(q)$. Figure 1.4 shows the results of the simulations for an input $q$-distribution according to the IMF; Fig. 1.5 the results for a flat input $q$-distribution; Fig. 1.6 shows the same for an input $q$-distribution $\left(\propto q^{1.5}\right)$ according to Popov (Popova et al. 1982).

It should be pointed out here that the numbers of 'generated' and 'observed' systems are not meant to represent real numbers; only the shapes of the distributions and the relative numbers are considered. The actual numbers of systems that were used in the simulations are a compromise between CPU time and statistical noise. Generated were 10000 systems. The simulations programme went on generating systems, until 3000 were 'observed' (often this meant that more than 10000 systems were generated). Remarks about the absolute numbers, and the rate of duplicity of 


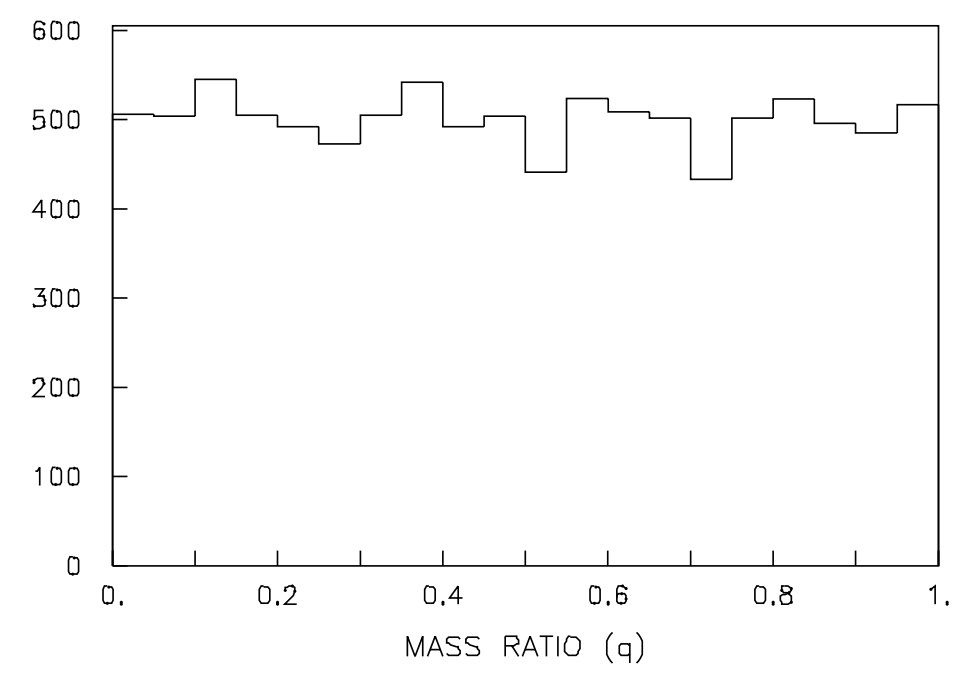

(a)

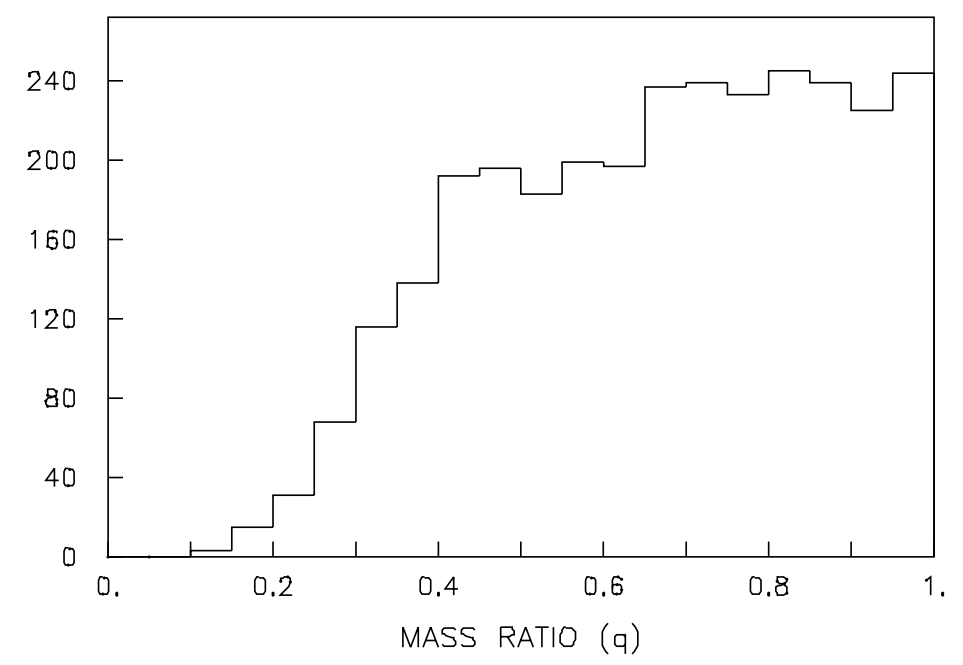

(b)

Figure 1.5: Simulations with $q$-distribution flat. (a) Generated $q$-distribution. $q-$ Distribution obtained for simulated observations.

stars are made in the discussion, section 1.6.7.

The 'observed' $q$-distributions, obtained from the simulations with an input distribution $\Phi(q)$ according to the IMF, agree very well with the actually observed $q$-distribution of the WDS systems (not corrected for selection effects) as given by Vereshchagin et al. $(1987,1988)$. This is illustrated by Fig. 1.4.b, in which the $N(q)$ distributions from simulations (solid line) and from the WDS (dashed line) have been super imposed. The histogram for the observed systems is the same as distribution nr. 3 in Fig. 1.1.a, for systems with primary magnitudes $m_{\text {prim }}<7^{m}$. The other input $q$-distributions yield simulated observed distributions (with PDFs $\phi_{s}(q)$ ) which cannot be brought in agreement with the $\phi(q)$ curves from the WDS, as shown by Figs. 1.5 and 1.6).

Figure 1.7 shows the good agreement between the distribution of angular separations $\phi_{s}(\rho)$ from the simulations of Fig. 1.4 (solid line), and the $\phi(\rho)$ distribution 


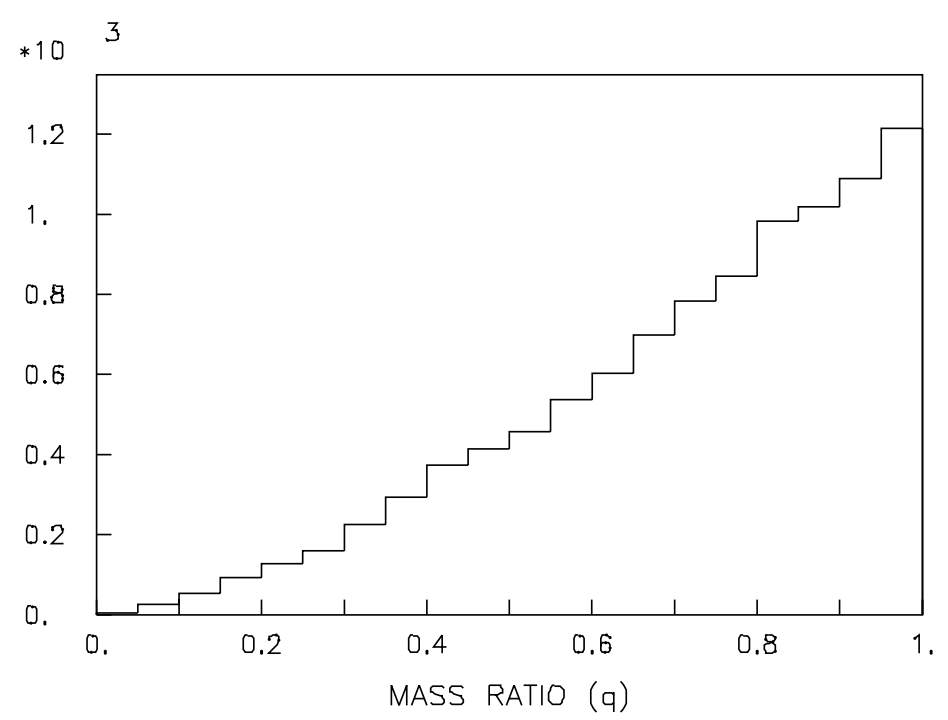

(a)

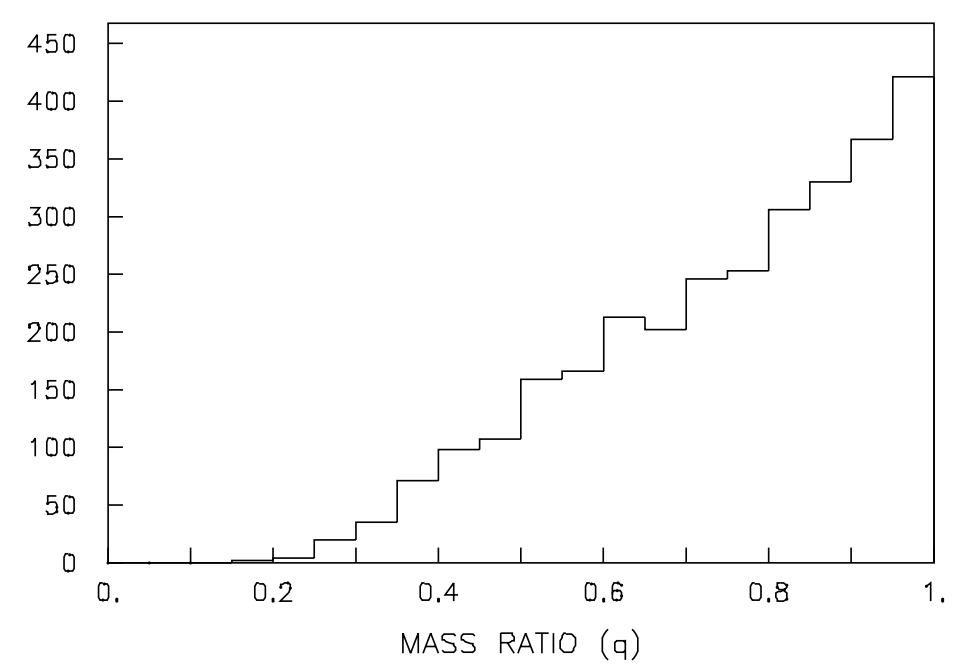

(b)

Figure 1.6: Simulations with PDF $\Phi(q)$ according to Popov (Popova et al. 1982). (a) Generated $q$-distribution. (b) $q$-Distribution obtained for simulated observations.

derived from the WDS (dashed line). Although systems were generated with semimajor axes $a$ as small as $1.4 R_{\text {Roche, none with } a<300 R_{\odot}}$ were 'detected' in the simulations. The simulated 'observed' distributions basically cover the same range in separations as the actually observed sample in the WDS.

The simulations with flat or rising 'input' distributions, as proposed by Popova et al. (1982), show that these distributions would be revealed in the observed distributions (Figs. 1.5 and 1.6). Since they are not, we must conclude that the real $q$-distribution of the stars in our galaxy does agree with an IMF, at least for $q>0.35$. Below that value for $q$ selection effects begin to play a major part. 


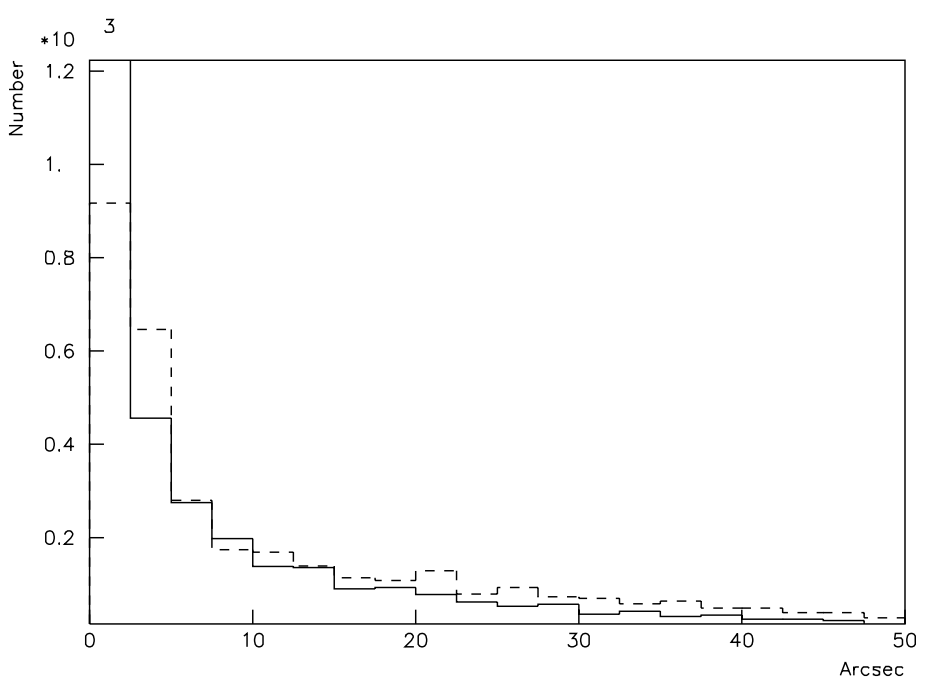

Figure 1.7: Distributions of angular separations $\rho$ from simulations (solid line) and from the WDS (dashed line) superimposed.

\subsection{Visual binaries with computed orbits}

According to Trimble and Walker (1986), the observed $q$-distribution derived from the OVB, is difficult to explain in terms of an IMF for the masses of the secondaries. Their tabulated $q$-distribution is graphically represented in Fig. 1.9.b. Trimble and Walker indicate that, if the peak were to be explained in terms of a real distribution according to the IMF, there should be another 30000 visual binaries in the sky with $0.3<q<0.9$. These binaries, apart from their $q$-values, would need to have about the same physical properties as the systems in the OVB, i.e., they would have to be in about the same magnitude range as the stars in the OVB, and thus observable.

\subsubsection{Selection effects in the OVB}

Since the OVB is a specially selected subset of the WDS, one expects that it is affected much more severely by selection effects than the WDS itself. I attempted to assess these selection effects in quantative terms by various procedures. In my attempts, I again applied numerical simulations, in which I tried to model the selection effects in relatively simple quantative terms, in ways analogous to the modelling of the selections effects in the WDS. However, all these attempts failed, which to me was an indication that, for the OVB, a simple and straight forward modelling of the selection effects is not possible.

To give an indication of the problems that have to be addressed: in addition to the selection effects mentioned in Sect. 1.3.3 for the WDS, there will, for the OVB, be a selection against longer periods, as a system has to be observed for a certain amount of time to accumulate the data which allow the computation of an orbit with a reasonable degree of accuracy. The OVB, however, is a very inhomogeneous mixture of systems. Some have been observed for several centuries, others for only a number of decades; some have been observed in eccentric orbits near periastron (such that their orbits could be determined, even though they have very long revolution periods), 
others near apastron. The selection effect against longer periods is a complex mixture of the separation of the components, of observational time spans, orbital eccentricities and orbital phase during the past few centuries, the decision of an observer that he has gathered enough data to determine an orbit, etcetera.

I could not find any tractable mathematical form to model this complex and inhomogeneous mixture of selection effects. As it turns out, there is even no way to determine up to what orbital period - if any - the OVB can be considered to be a complete statistical sample. Yet one would like to find a way to decide whether the $q$ distribution found for the systems in the OVB could, in principle, still be compatible with the IMF like distribution which was found for the secondaries of the binary systems in the WDS.

A possible solution of the problem poses itself if one realizes that the $q$-distribution found from the OVB is very much determined by the way in which observers have selected the visual binary systems for which they decided to determine an orbit. The choice of the observers is determined by a procedure which I will call 'biased sampling', which appears to provide a possible (and probable) way to explain the $q$-distribution derived by Trimble and Walker. This procedure is considered in the following section.

\subsubsection{Biased sampling}

There are several physical quantities which make it more likely for some binary systems to have their orbits determined than others. For instance, the period of the system may not exceed the time span of the observations by too much, because then the components will not have shown enough (angular) motion. For their periods to be not too long, the components have to be relatively close to each other, which means that their angular separations must be small (of the order of a few arc seconds). In order to be properly observable, the components of a system should then not differ too much in brightness, because a faint component cannot be observed when it is close, in angular separation, to its bright companion. Also, the separation and position angle of a faint component cannot be determined as accurately as they can for a brighter component.

In view of the above, it seems reasonable to assume that, in the subset of systems with small angular separations, the magnitude difference of the components is the dominating criterion for an observer in selecting a system for which he will determine an orbit. Also, one must assume that observers do not first search the entire sky for the 'best' observable binaries; it is more likely that they pick the 'good' ones (to determine an orbit for) as they come along in searches for visual binary stars. This choice of 'good', rather than the 'best' systems from the binaries in the entire sky, can in simple mathematical terms be described as follows.

A sample of $N$ 'good' elements, rather than the $N$ 'best', can be obtained from a population of $\mathcal{N}$ elements, by dividing the population into $N$ sub-samples of $n=$ $\mathcal{N} / N$ elements. When from each sub-sample of $n$ elements the 'best' element is taken, the new sample contains $N$ 'good' elements of the original population, since the 'best' element in one sub-sample may be worse than the second, third or even fourth best in another sub-sample. We may consider $\mathcal{N}$ the number of observable visual binaries 


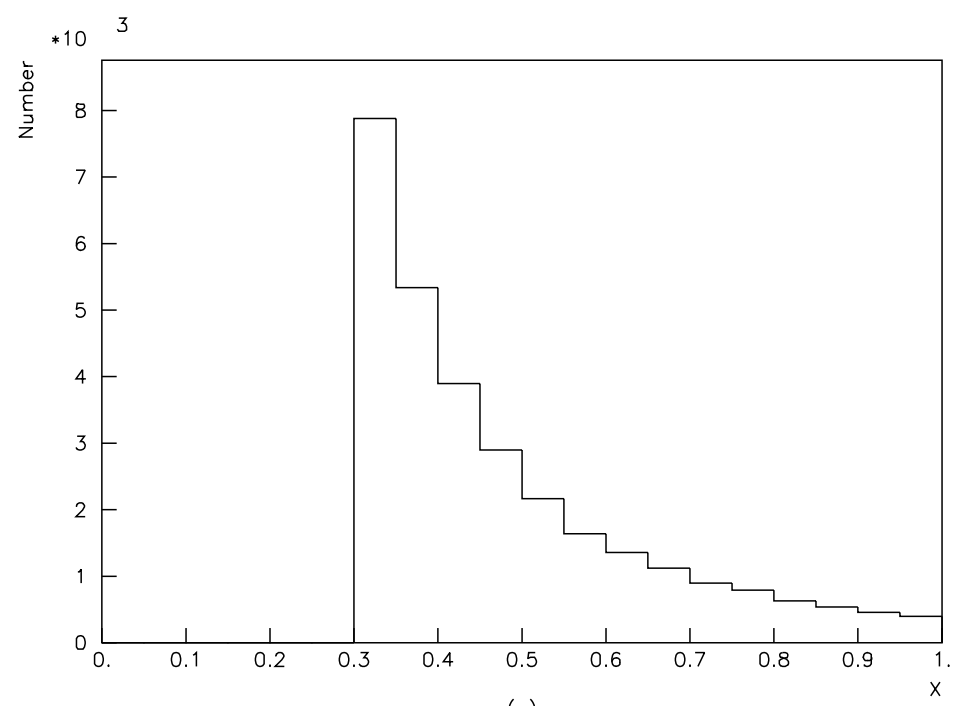

(a)

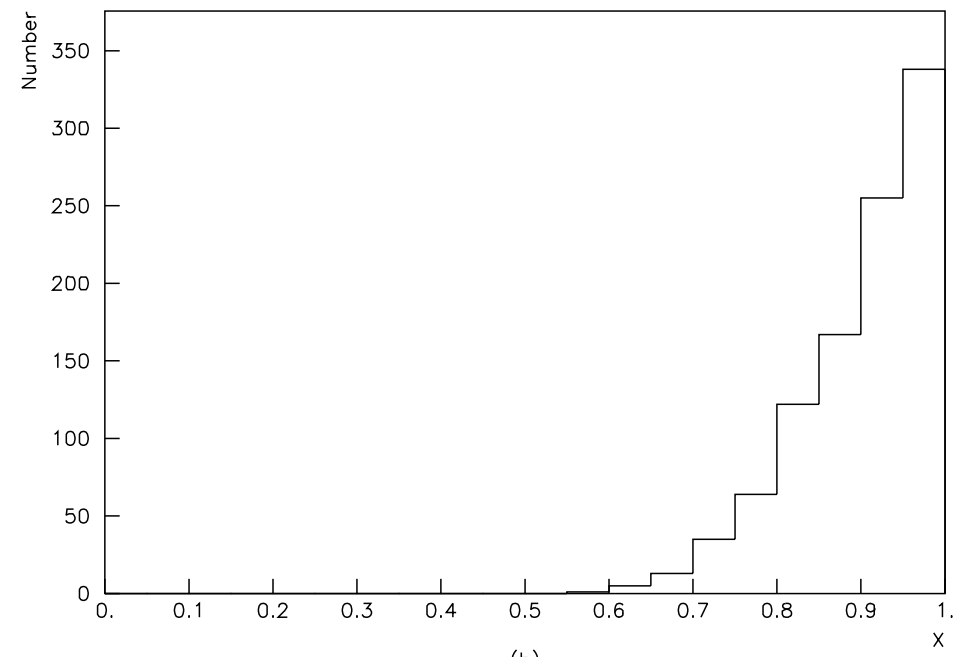

(b)

Figure 1.8: Illustration of the statistical method employed in section 1.5 to draw a biased sample from a larger population. (a) The parent population of 30000 elements, distributed with PDF $\phi(x) \propto x^{-2.7}$ in the interval $0.3<x<1$. (b) Distribution of the resulting sample of 1000 elements, showing a pronounced peak in the distribution at $x=1$.

in the entire sky, and $N$ the number of systems in the catalogue (OvB).

To illustrate this, figure 1.8 shows what happens when a sample of 1000 elements is drawn in this way from a population of 30000 elements, distributed according to $\operatorname{PDF} \Phi(x) \propto x^{-2.7}$, for $x$ in the interval $(0.3,1)$. (Here $\mathcal{N}=30000$, and the sample of $N=1000$ elements results from selecting from 1000 sub-samples of $n=\mathcal{N} / N=30$ elements those which have a value closest to $x=1$.) The figure shows that the distribution of the sample of 1000 elements exhibits a pronounced maximum near $x=1$, quite similar to the $q$-distribution found from the ovB by Trimble and Walker.

Although this is no proof, the similarity of the two distributions suggests that: (i) the $q$-distribution of the OVB systems might be determined mainly by a sampling procedure in which systems are selected with a preference for bright companion stars; 


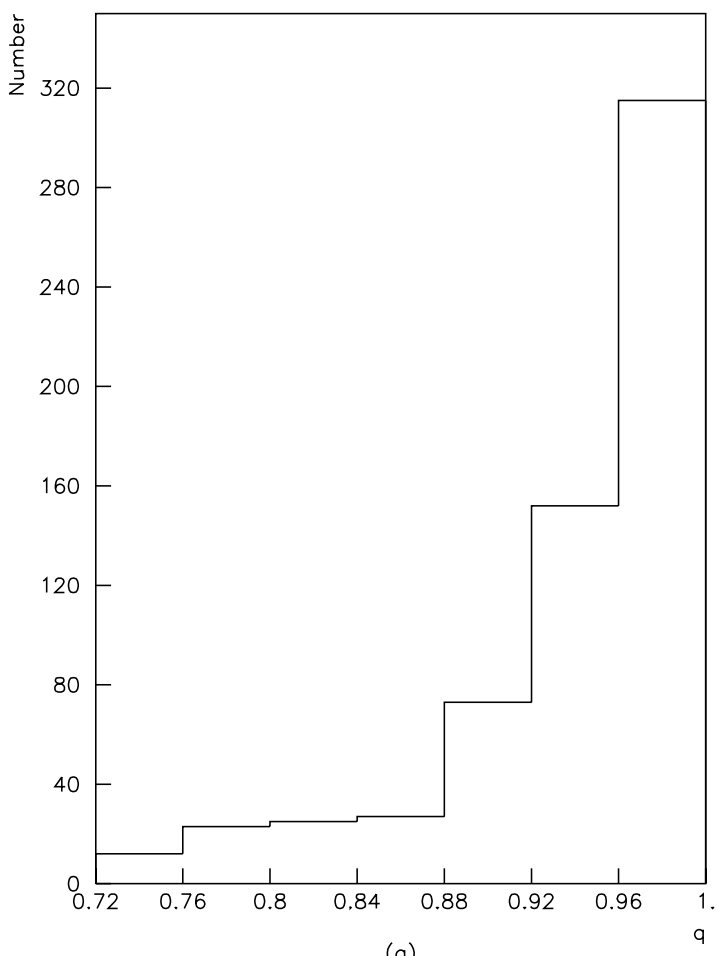

(a)

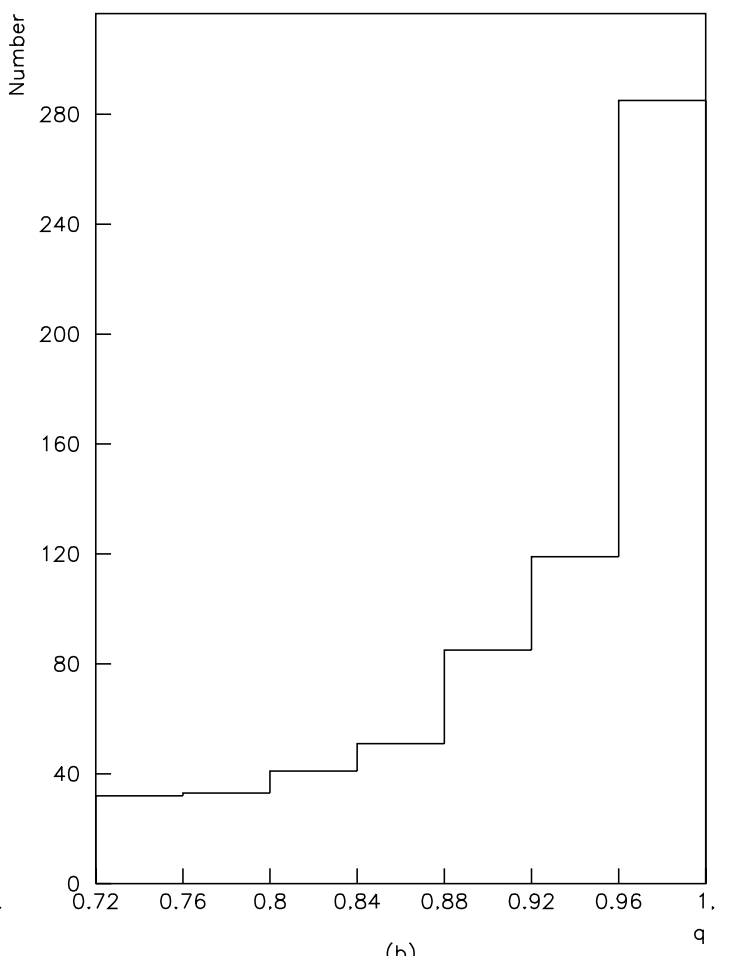

(b)

Figure 1.9: (a) $q$-Distribution of a sample of 650 systems, drawn from the 8862 systems in the WDS with primary magnitudes $m_{\text {prim }} \leq 8.5^{m}$, according to the statistical method decribed in section 1.5. The distribution compares well with the $\phi(q)$ distribution of 646 systems in the OvB (panel $b$ ). (b) The peak in the $\phi(q)$ distribution from the OVB (Trimble and Walker, 1986).

(ii) the observed $q$-distribution of the OvB could still be compatible with a real $q-$ distribution according to the IMF.

The sampling procedure was repeated using $N$ sub-samples of unequal sizes $\left(n_{i}\right.$ ranging from 1 to 60 , according to different distributions), which all yield a strong peak near $q=1$, but different 'tails' as $q \rightarrow 0$. However, the tails are not very significant with respect to the peak.

\subsubsection{The relation between OVB and WDS systems}

It was found possible to derive a sub-sample from the WDS, with the statistical method described above, which yields a $q$-distribution similar to the one found from the OVB, as follows. The peak in the $q$-distribution between $q=0.72$ and $q=1$ for the OVB systems, consists of 646 systems. When from the 8,862 systems in the WDS with magnitudes $m_{\text {prim }} \leq 8.5^{\mathrm{m}}$ a sub-sample of 650 systems is drawn in the way described above, the $q$-distribution of this sample shows a $q$-distribution similar to the distribution found from the OVB. This is shown by Fig. 1.9.a.

How can it be that we arrive at a sub-sample with the right number of systems, with about the correct observed $q$-distribution, from a parent population which con- 
tains 8862 systems, and not the 30000 systems with $0.3<q<0.9$, deemed necessary by Trimble and Walker if the $q$-distribution would follow the IMF? A statistical investigation of the WDS shows that the distribution of secondary magnitudes has a maximum for $m_{\mathrm{sec}}=11-12^{m}$. If we assume that the WDS is complete for secondaries with $m_{\mathrm{sec}} \leq 11^{m}$, then the observations of systems with primary magnitudes up to $8.5^{m}$ are only complete for $\Delta m \leq 2.5^{m}$. When a main-sequence mass-luminosity relation is assumed, a relation

$$
q=10^{-\Delta m / 10}
$$

can be deduced (Halbwachs 1983). According to this relation, a $\Delta m$ of $2.5^{m}$ corresponds to $q=0.6$. With a $q$-distribution proportional to $q^{-2.7}$, there is a factor 3.2 between the number of elements found in the interval $(0.6,1)$ and the number found in the interval $(0.3,1)$. If we multiply the 8862 systems in the WDS, with primary magnitudes $m_{\text {prim }} \leq 8.5^{m}$, with this incompleteness factor, we find that there will be about 28000 systems with $0.3<q<1$, which is very close to the 30000 systems that are required according to Trimble and Walker, if the real $q$-distribution of visual binaries follows the IMF.

The assumption that observers favour systems with components of nearly equal magnitudes to determine orbits for, is supported by Fig. 1.10. Figure 1.10.a. shows the scatter diagram of primary versus secondary magnitudes for all 73,160 systems in the WDS. Figure 1.10.b. shows the same diagram for all 847 systems in the OVB. It is obvious that the systems in Fig. 1.10.b. are concentrated toward the line $m_{\mathrm{sec}}=m_{\text {prim }}$, whereas the systems in the WDS show a much greater spread in secondary magnitudes.

\subsection{Discussion}

\subsubsection{Minimum detectable angular separation}

The major selection effect, dominating observations of visual binary stars, is the minimum detectable angular separation $\rho_{\min }$, as a function of magnitude difference of the components $\Delta m$. The maximum angular separation $\rho_{\max }$, at which an optical pair is accepted as a physical binary, is not a selection effect, but a criterion, set by visual binary observers. This criterion, since it is used in the compilation of the WDS, has simply been copied into the simulation programme. The maximum value for $\Delta m$ will be discussed later.

The minimum detectable angular separation $\rho_{\text {min }}=f(\Delta m)$ was in this paper derived from observations. This selection effect is due to the combination of the observers eye, and his telescope (the larger part of the systems in the WDs have been observed visually with telescopes with apertures between 50 and 90 centimeters). Although it is quite obvious that the eye, as a detector, will behave in about the way as suggested by the $\rho_{\min }=f(\Delta m)$ relation, one would like to have a more quantative measure, independent from the observations themselves. This quantative measure, where also the input of the system is under control (i.e., the light of the binary star that enters the telescope) could be provided by research into human perception. This kind of research is performed at several institutes around the world. However, it has been pointed out to me by F.J.J. Blommaert of the Institute for Perception Research in Eindhoven, The Netherlands (private communication) that the precise 


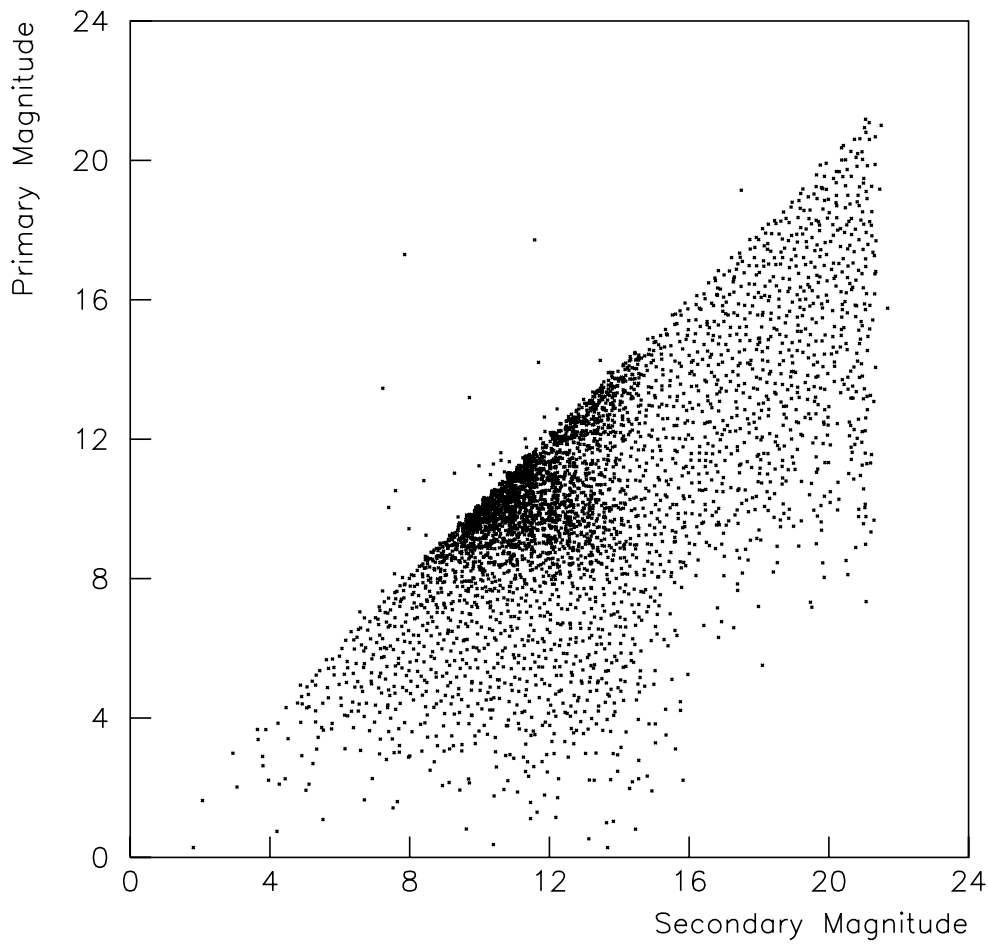

(a)

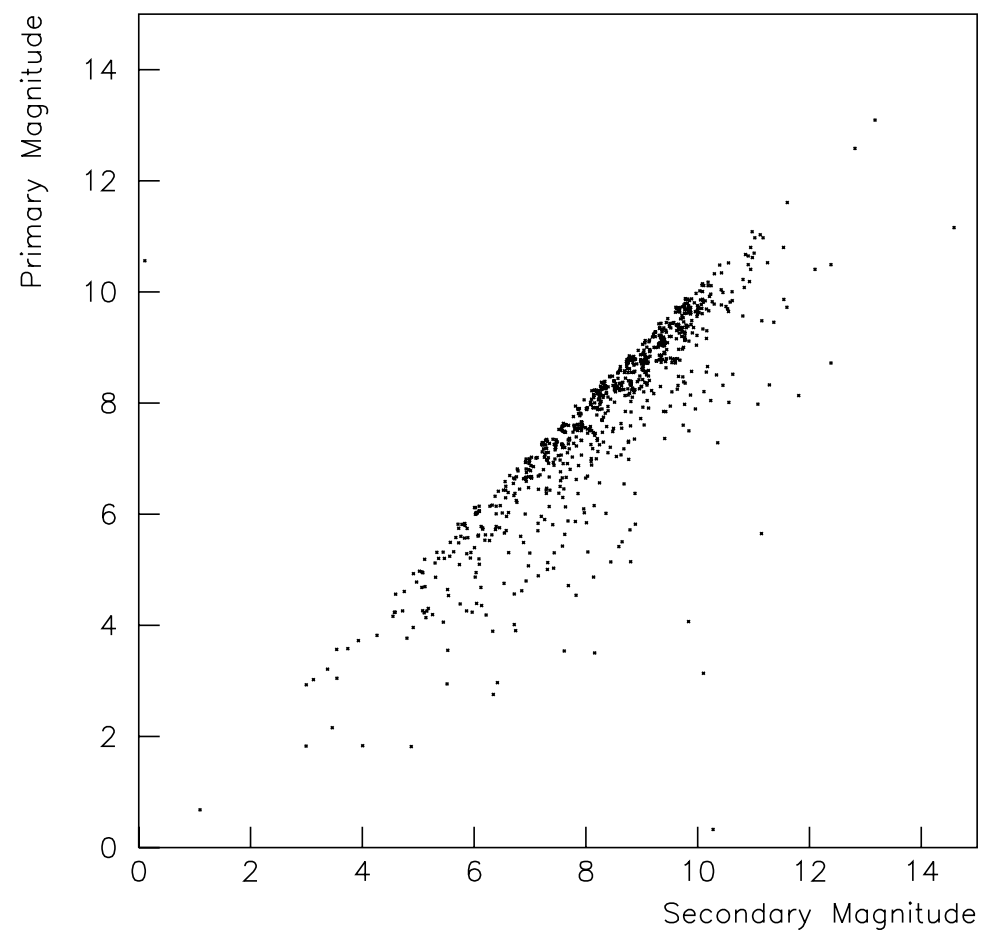

(b)

Figure 1.10: Scatter diagrams of primary vs. secondary magnitudes for $(a)$ the WDS and $(b)$ the ovB. (Notice that the maximum magnitude in panel $a$ is $24^{m}$, while in panel $b$ it is $\left.15^{m}.\right)$ 
perceptional conditions, of observing visual binaries at the eyepiece of a telescope, have not been investigated so far. As already indicated by Öpik (1924) and Heintz (1969), the line in the $\Delta m-\log \rho$ diagram should be a strip of some width, in which the capability of the eye to detect a secondary decreases gradually. However, as I have shown, the crude aproximation with the proposed line in the $\Delta m-\log \rho$ diagram yields useful results.

\subsubsection{Magnitude difference}

In section 1.3.3 it was reported that the maximum near $q=0.35$ in the observed $q$ distribution presented by Vereshchagin et al. $(1987,1988)$ could only be reproduced in the simulations when a maximum magnitude difference $\Delta m_{\max }$ was incorporated. What needs to be established is whether this maximum $\Delta m$ is a selection effect, or if it is due to the physical nature of binary stars.

One physical limit for a maximum $\Delta m$, or rather a minimum value for the massratio, can be deduced from stellar life times. Iben (1965) presents data for mainsequence life-times, and life-times for pre-main-sequence contraction phases for stars of different masses. From these data, it can be deduced that the mass-ratio for two main-sequence stars cannot be much smaller than $q=0.2$, since below that value the less massive component is still in the contraction phase, while the more massive component is evolving away from the main-sequence already.

This lower limit for $q$ is smaller than the value of $q=0.35$ at the turnover point in the diagrams presented by Vereshchagin et al. Investigation of the WDS shows that the maximum magnitude difference observed for systems with fainter primaries becomes smaller (section 1.5, Figs. 1.3.a and 1.3.b). This behaviour can be expressed as:

$$
m_{\text {prim }}+\Delta m_{\max }=11^{m}
$$

From the systems in the WDS with bright primaries it is clear that magnitude differences up to $\Delta m=10$ do occur frequently. So, for systems with fainter primaries, it must be concluded that systems with larger values for $\Delta m$ have not been observed due to selection effects. Through relation (1.3) the effect of $\Delta m_{\max }$ for various primary magnitudes on the minimum value of $q$ can be assessed. For systems with primary magnitude $m_{\text {prim }}=7^{m}, \Delta m_{\max }=4^{m}$, which means $q_{\min }=0.4$. This allows us to conclude that the turnover at $q=0.35$ in the distributions presented by Vereshchagin et al. $(1987,1988)$ is indeed due to selection effects.

In a separate paper on the statistical properties of spectroscopic binaries, it will be shown that for F, G,K-type systems the real $q$-distribution (i.e. corrected for selection effects) only departs from an IMF-like behaviour for $q<0.25$.

\subsubsection{Supportive evidence}

Supportive evidence for a distribution of secondary masses according to the IMF for visual binaries, is presented by Halbwachs (1983). Halbwachs considered the statistics of visual binary stars in the Yale Catalogue of Bright Stars (Hoffleit and Jaschek 1982). After a careful investigation of the selection effects, Halbwachs arrives at a mass-ratio distribution according to the IMF. 
Although the binaries in the Yale Catalogue are also in the WDS, the investigations of Halbwachs may be regarded as independent evidence, for the binaries in the Yale Catalogue are not the result of a systematic search for binaries. Rather, they are bright stars which happen to be visual binaries. Apart from the fact that they are bright stars, the visual binaries in the Yale Catalogue comprise a relatively unbiassed sample.

\subsubsection{The peak near $q=1$}

It has been shown in section 1.5 that the pronounced peak in the $q$-distribution derived from the OVB (Fig. 1.1.b), can be attributed to a sampling selection effect. The four distributions published by Vereshchagin et al. $(1987,1988)$ were derived from the IDS, for systems with primary magnitudes $m_{\text {prim }} \leq 5,7$ and $9^{m}$, and for all systems (Fig. 1.1.a). All curves show a maximum at $q=0.35$, which has been explained in section 1.6.2 to be due to selection effects. Three of the four curves show a second maximum in $\phi(q)$ for $q=1$. The second maximum is not present in the curve for systems with $m_{\text {prim }} \leq 7^{m}$, which Vereshchagin et al. consider the most complete and unbiassed sample. For systems with primaries up to $m_{\text {prim }}=9^{m}$, the selection effect against systems with larger values for $\Delta m$, and thus against systems with smaller $q$-values, becomes more obvious (section 1.6.2, Eq. 1.4). This accounts for the larger fraction of systems with $0.6<q<1$ in this sample.

Vereshchagin et al. do not give an account of the second peak in the $q$-distribution for systems in Fig. 1.1.a with primary magnitudes $m_{\text {prim }} \leq 5^{m}$ (distribution nr. 4). The $d N(q) / d q$ scale in Fig. 1.1.a is logarithmic, which means that the second peak reaches $10 \%$ of the value at $q=0.35$.

To establish the reality and significance of the secondary maxima in $\phi(q)$ near $q=1$ further investigation is required.

\subsubsection{Other 'generated' distributions}

In this paper, I restricted myself to simulations with different input $q$-distributions. The distributions of other parameters, such as the semi-major axis and the eccentricity of the orbit, which also may be expected to be significant, were left unchanged. However, some test runs with other input distributions for semi-major axis $a$ and for eccentricity $e$ have been made.

The PDF $\Phi(a)$ has been adapted to yield a flat $N(a)$ distribution. The $\rho$ distribution that resulted from these simulations was no longer in agreement with the distribution of angular separations with $\operatorname{PDF} \phi(\rho)$ derived from the WDS. However, the flat (input) distribution of semi-major axes had no significant effect on the simulated observed $q$-distribution.

The simulations were also performed with eccentricity $e=0.5$, instead of the circular orbits $(e=0)$ that were assumed in the simulations presented in this paper. This had no significant influence on the 'observed' distributions for $q$ and $a$. 


\subsubsection{Semi-major axis}

The real distribution of semi-major axes was assumed to have a PDF $\Phi(a)$ proportional to $1 / a$. Halbwachs (1983) discusses the features of $\phi(a)$, with respect to the findings of other authors. The distribution of angular separations with PDF $\phi_{s}(\rho)$, found from the simulations with $\Phi(a) \propto 1 / a$, is in good agreement with $\phi(\rho)$ found for systems in the WDS.

\subsubsection{Rate of duplicity}

We have found that for $q>0.35$ the masses of secondaries are distributed according to the IMF. For $q<0.35$ the behaviour of the $q$-distribution cannot be established because of selection effects. Therefore, we cannot know for which value of $q$ the distribution departs from the power law PDF $\Phi(q) \propto q^{-\alpha}$. We know that the $q-$ distribution should flatten for some smaller value of $q$, because for $q \rightarrow 0, q^{-\alpha}$ tends to infinity. According to Scalo (1986) the IMF flattens for masses $<0.3 M_{\odot}$.

From the studies by Vereshchagin et al. (1987) we may decide that the WDS contains all observed systems with $m_{\text {prim }}<7^{m}$ and $q>0.35$. There are 3,255 systems in the WDS with $m_{\text {prim }}<7^{m}$. If we assume a homogeneous distribution of stars in space, then we may expect that there are 18,200 stars with magnitudes $<7^{m}$, based on the 9,110 stars with magnitudes $<6.5^{\mathrm{m}}$ in the Yale Catalogue of Bright Stars (Hoffleit and Jaschek 1982). If the WDS contains all visual binaries up to the seventh magnitude, this means that $18 \%$ of all observable stars are visual binaries. In a study of the main-sequence binary systems in the Yale Catalogue of Bright Stars, Halbwachs (1983) finds that about $40 \%$ of the stars are visual binaries with $q>0.2$. This percentage is (for a $q$-distribution according to the IMF) in agreement with $18 \%$ binaries with $q>0.35$.

For late type spectroscopic binaries (F,G,K-type) (Hogeveen 1991, this thesis Chapter 3) it is found that the $q$-distribution also adheres to the IMF, for $q>$ 0.25. However, for spectroscopic binaries it can be shown that the turnover in the distribution for $q=0.25$ is due to flattening of the real $q$-distribution. If we assume the same turnover in the real distribution of the visual binaries, then there are more binaries than we are able to observe. The real number of binaries would be about 3.4 times the observed number, yielding a visual binary frequency of about $60 \%$.

Earlier in this discussion (section 1.6.2) it was argued that a binary with two main-sequence components is unlikely to have a mass-ratio much less than $q=0.2$. For a $q$-distribution wich follows the IMF for $q>0.2$ and is flat for $0<q<0.2$, we find that $83 \%$ of the stars are in visual binaries.

Since visual binaries and spectroscopic binaries are different sub-groups of the binary population, it is not at all clear whether the turnover at $q=0.25$, found for late type spectroscopic binaries, applies to the visual binaries. Therefore, the rates of duplicity presented here have to be regarded as crude estimates. 


\subsection{Conclusions}

The selection effects that govern the observations of visual binary systems have been investigated, in order to gain insight in the real distribution of mass-ratios of unevolved systems. It was found that the real $q$-distribution agrees with the Initial Mass Function, for $q>0.35$. The turnover at $q=0.35$ in the observed distribution must be attributed entirely to selection effects. Therefore it is difficult to establish the rate of duplicity. It is estimated that $36-60 \%$, and possibly as much as $83 \%$ of the stars are in binary systems.

It is shown that a peak in the $q$-distribution near $q=1$, as reported by some authors, is likely to be entirely due to selection effects. The observed peak can be explained in terms of a sampling selection effect.

Mass-ratio distributions derived from the WDS might show a second maximum near $q=1$. This maximum is, however, much less pronounced as the maximum near $q=1$ found for binaries in the OVB, and its reality remains to be established.

\section{Acknowledgements}

I wish to acknowledge stimulating discussions with E.P.J. van den Heuvel. I also wish to acknowledge the useful comments provided by J.A. van Paradijs, Jacqueline Coté and Wim Penninx. I wish to thank Dr. F.J.J. Blommaert of the Instituut voor Perceptie Onderzoek (institute for research into human perception), Eindhoven, The Netherlands for his comments on the perceptional conditions that may exist when visual double stars are observed at the eyepiece of a telescope. I gratefully acknowledge the use of the computer facilities of and support from NIKHEF-H (Netherlands Institute for Nuclear and High Energy Physics, section H) in Amsterdam. I wish to thank Dr. Halbwachs of the Centre de Données Stellaires of the Observatoire Strassbourg for providing me with magnetic tape versions of The Washington Double Star Catalogue (WDS) and the Fourth Catalogue of Orbits of Visual Binary Stars (OVB). Figure 1.1.a has been reproduced from Vereschagin et al. (1987) by courtesy of The American Institute of Physics; Fig. 1.1.b has been reproduced from Trimble and Walker (1986) by courtesy of Kluwer Academic Publishers. My work is supported by the Netherlands Foundation for the Advancement of Research, grant 782-371-026.

\section{Appendix}

\section{A.1 Introduction}

A binary system is fully described by seven parameters (Sect. 1.2). The distance was introduced as an important eighth parameter. From these eight parameters, other parameters of interest can be derived, such as the period, the apparent magnitude of the components, the angle of separation, etc.

For a sample of binaries all these parameters will show statistical distributions. Section 1.A.2 gives a brief explanation about how a distribution (a Probability Density Function or PDF) is turned into a random variate. Later sections give an account of how a synthetic sample of binaries is generated from random variates for the eight 
basic parameters, and of the formulae that were used to derive other parameters from the basic ones.

\section{A.2 Producing random variates}

An extensive and complete discussion of how a random variate is reproduced from a Probability Density Function is given by Banks and Carson (1984).

Given a process with a PDF $f(x)$ in the interval $(a, b)$, a Cumulative Distribution Function (CDF) $F(x)$ can be defined as:

$$
F(x)=\int_{a}^{x} f\left(x^{\prime}\right) d x^{\prime}
$$

with $x$ in $(a, b)$, while $F(a)=0$ and $F(b)=1$.

When we now write Eq. (1.5) as $F(X)=R$, we may consider $X$ in the interval $(a, b)$ the random variate we wish to produce, and $R$ a (real) number in the interval $(0,1)$. The inverse function

$$
X=F^{-1}(R)
$$

will produce random variate $X$ with a PDF $f(x)$ when $R$ is a random number in the interval $(0,1)$.

This technique of producing random variates from PDF's was used for all 'generated' parameters in the computer programme.

\section{A.3 Reproducing a normal distribution}

Banks and Carson (1984) present several methods by which a normal distibution can be generated. The method employed in the present simulations is not the most efficient, but it is elegant and easy to programme. From two random numbers $R_{1}$ and $R_{2}$, two independent numbers $Z_{1}$ and $Z_{2}$ can be calculated, which are distributed according to a normal distribution with mean zero and $\sigma^{2}=Z_{i}^{2}$. The algorithm by which $Z_{1}$ and $Z_{2}$ are calculated from $R_{1}$ and $R_{2}$ is:

$$
\begin{aligned}
& Z_{1}=\left(-2 \ln R_{1}\right)^{\frac{1}{2}} \cos 2 \pi R_{2} \\
& Z_{2}=\left(-2 \ln R_{1}\right)^{\frac{1}{2}} \sin 2 \pi R_{2}
\end{aligned}
$$

\section{A.4 Generated parameters}

The eight basic parameters that fully describe a binary system (listed in Sect. 1.3.1) were generated from random variates as described below.

\section{Primary and secondary mass}

The primary mass was in all cases deduced from the Initial Mass Function (IMF):

$$
f(M) \propto M^{-\alpha},
$$

where $\alpha$ is a positive real number. 
From the PDF $f(M)$, with mass $M$ in interval $\left(m_{1}, m_{2}\right)$, a CDF can be derived, obeying the conditions of formula (1.5):

$$
F(M)=\int_{m_{1}}^{M} f(m) d m
$$

with $F\left(m_{1}\right)=0$ and $F\left(m_{2}\right)=1$.

Following the procedure of section 1.A.2, the random variate $X$ obeying PDF $f(M)$ is produced from random number $R$ in interval $(0,1)$ by:

$$
X=F^{-1}(R)=\left(R C+m_{1}^{(1-\alpha)}\right)^{1 /(1-\alpha)}
$$

$C$ is determined by $\alpha, m_{1}$ and $m_{2}$ as:

$$
C=m_{2}^{(1-\alpha)}-m_{1}^{(1-\alpha)}
$$

The mass of the secondary is calculated from the primary mass and the mass-ratio. The latter is deduced from an imposed $q$-distribution. The mass-ratio is defined as $q=M_{2} / M_{1}$. When the PDF of the mass-ratio is also assumed to behave like a power law, then the CDF and random variate producing algorithm are found along the same lines as the primary mass distribution:

$$
F(q)=\int_{q_{0}}^{q} f\left(q^{\prime}\right) d q^{\prime}
$$

with $F\left(q_{0}\right)=0$ and $F(1)=1 ; q$ is in the interval $\left(q_{0}, 1\right)$.

With a distribution behaving like Eq. (1.9), one cannot have the lower boundary equal to zero, because there $f(q)$ would tend to infinity. The random variate $X$ with PDF $f(q)$ is now found to be:

$$
X=F^{-1}(R)=\left(R C+q_{0}^{(1-\alpha)}\right)^{1 /(1-\alpha)}
$$

$C$ this time being determined by $\alpha$ and $q_{0}$ as:

$$
C=1-q_{0}^{(1-\alpha)}
$$

\section{Semi-major axis}

Throughout this paper the relative orbit of the binary system is considered. The distribution of semi major-axes $a$ is assumed to be proportional to $1 / a$ (section 1.3.2). With $a$ in the interval $\left(a_{1}, a_{2}\right)$ the CDF for the distribution of semi major-axes becomes:

$$
F(a)=\int_{a_{1}}^{a_{2}} f\left(a^{\prime}\right) d a^{\prime}
$$

with $F\left(a_{1}\right)=0$ and $F\left(a_{2}\right)=1$. Random variate $X$ with PDF $f(a)$ is then found from:

$$
X=F^{-1}(R)=a_{1} e^{R C}
$$

$C$ is determined by $a_{1}$ and $a_{2}$ as:

$$
C=\ln a_{2}-\ln a_{1}
$$




\section{Inclination of the orbital plane}

It was assumed that the orbital plane of the generated binary systems could have any orientation in space. This means that the normal vector, perpendicular to the orbital plane, has an equal probability to be found pointing in any portion of solid-angle $d \omega$, or: $P(\omega) d \omega=$ constant. Solid angle $\omega$ can be expressed in terms of spherical coordinates $\theta$ and $\phi$ as:

$$
d \omega=\sin \theta d \theta d \phi
$$

In terms of 'observational' parameters, $\theta$ is equal to the inclination $i$. If we now define the probability $P(i, \phi)$ for the normal vector to have coordinates $i$ and $\phi$ in the intervals $i, i+d i$ and $\phi, \phi+d \phi$ equal to $P(\omega)$ :

$$
P(i, \phi) \operatorname{did} \phi=P(\omega) d \omega
$$

we find $P(i, \phi)=P(\omega) \sin i$, or:

$$
P(i, \phi) \propto \sin i
$$

So for the inclination, we have a PDF $f(i) \propto \sin i$, with $i$ in interval $(0, \pi)$. We can now define CDF $F(i)$ as:

$$
F(i)=1 / C \int_{0}^{i} \sin i^{\prime} d i^{\prime}
$$

such that $F(0)=0$ and $F(\pi)=1$.

The inverse of $F(i)$ gives us the formula generating the random variate $X$ with PDF $f(i)$ :

$$
X=F^{-1}(R)=\arccos (1-R C)
$$

$C$ being determined by: $C=\cos 0-\cos \pi=2$.

\section{Longitude of periastron}

In general, the (relative) orbit of a binary system is an ellipse with eccentricity $e \neq 0$. The longitude of periastron $\omega$ is measured in the orbital plane (cf. Batten, (1973)) and it gives the orientation of the major axis in the orbital plane with respect to the line of nodes.

When we allow this angle to assume any value $0 \leq \omega<\pi$ (because of the ambiguity in direction one does not need to go to $2 \pi)$, the PDF $f(\omega)=$ constant.

The CDF can thus be defined as:

$$
F(\omega)=1 / C \int_{0}^{\omega} c d \omega^{\prime}
$$

with $F(0)=0$ and $F(\pi)=1$. When we have $c=1$, we find for the random variate generating formula:

$$
X=F^{-1}(R)=R C
$$

with $C=\pi$. 


\section{Distance}

The distribution of stars in space was assumed to be homogeneous. This means that the probability of finding a number of stars in a given volume of space is proportional to that volume.

At the location of the sun, the galactic disk has a thickness of about 400 parsec. In the simulations, it was possible to restrict the distances to values $R_{\max }<100$ parsec, which is entirely within the disc. With a homogeneous distribution of stars in space, the PDF $f(r)$ for finding a number of stars within a shell of radius $r$ and thickness $d r$, is proportional to $r^{2}$. The CDF then is (for $0<r<R_{\max }$ ):

$$
F(r)=1 / C \int_{0}^{r} r^{\prime 2} d r^{\prime}
$$

with $F(0)=0$ and $F\left(R_{\max }\right)=1$. The random variate $X$ with PDF $f(r) \propto r^{2}$ is then found from random numbers $R$ through:

$$
X=F^{-1}(R)=(3 C R)^{\frac{1}{3}}
$$

where $C=R_{\max }^{3} / 3$.

\section{A.5 Derived parameters: radii, luminosities, apparent magnitudes, and spectral types}

From the 'generated' parameters discussed in the previous section, the 'derived' parameters defined in Sect. 1.3.1 were obtained as described below.

The radii of the components of a binary system were determined from a mainsequence mass-radius relation derived from a table presented by Allen (1973):

$$
R=M^{0.786}
$$

This is an average relation that holds for main-sequence stars in the mass range of $0.1 M_{\odot}$ to $30 M_{\odot}$.

Luminosities were determined similarly from a mass-luminosity relation by Smith (1983):

$$
\begin{cases}L=0.23 M^{2.3} & \text { if } M<0.42 M_{\odot} \\ L=M^{4} & \text { if } M>0.42 M_{\odot}\end{cases}
$$

Although Smith indicates that this relation is valid for main-sequence stars in the mass range $0.1-10 M_{\odot}$, it has been applied to the mass range $0.1-30 M_{\odot}$.

The bolometric magnitudes were calculated from the luminosities of the components and the distance of the system to the observer. Because of our interest in Visual Binaries, these magnitudes were converted into visual magnitudes, by applying the bolometric corrections given by Allen (1973). Also we applied an extinction law, with an average extinction of 1.2 magnitudes per kpc.

$$
m_{v}=m_{\mathrm{bol}}-b c+m_{\mathrm{ext}}(d)
$$

The spectral types Sp1 and Sp2 of the components of a double star system were determined from black body temperatures, which were derived from the luminosities and the radii of the components. We used the effective temperature-spectral-type relation according to Hayes (1978). 


\section{References}

Aitken, R. G.: 1932, New General Catalogue of Double Stars, Carnegie Institution, Washington

Allen, C. W.: 1973, Astrophysical Quantities, The Athlone Press, University of London

Banks, J. and Carson II, J. S.: 1984, Discrete Event System Simulation, Prentice Hall, New Jersey

Batten, A. H.: 1973, Binary and Multiple Systems of Stars, Pergamon Press, Oxford

Halbwachs, J. L.: 1983, Astron. Astrophys. 128, 399

Hayes, D. S.: 1978, in A. G. Davis and D. S. Hayes (eds.), The H-R Diagram, pp 65-76, IAU Symposium 80, D. Reidel, Dordrecht

Heintz, W. D.: 1969, J. Roy. Astron. Soc. Canada 63, 275

Hoffleit, D. and Jaschek, C.: 1982, The Bright Star Catalogue, $4^{\text {th }}$ ed., Yale University Observatory, New Haven, U.S.A.

Hogeveen, S. J.: 1991, Astrophys. Space Sci., submitted

Iben Jr., I.: 1965, Astron. J. 141, 993

Jeffers, H. M., Van den Bos, W. H., and Greeby, F. M.: 1963, Index Catalogue of Visual Double Stars, Lick Observatory, Mount Hamilton

Kraicheva, Z. T., Popova, E. I., Tutukov, A. V., and Yungel'son, L. R.: 1985, Astrophysics $\mathbf{2 2}, 63$

Kuiper, G. P.: 1935a, Publ. Astron. Soc. Pac. 47, 15

Kuiper, G. P.: 1935b, Publ. Astron. Soc. Pac. 47, 121

Öpik, E.: 1924, Publ. Tartu 25, part 6

Popova, E. I., Tutukov, A. V., and Yungelson, L. R.: 1982, Astrophys. Space Sci. 88, 55

Poveda, A., Allen, C., and Parrao, L.: 1982, Astron. J. 258, 589

Pringle, J. E. and Wade, R. A.: 1985, Interacting Binary Stars, Cambridge University Press, London

Scalo, J. M.: 1986, Fundamentals of Cosmic Physics 11, 1

Smith, R. C.: 1983, The Observatory 103, 29

Trimble, V. and Walker, D.: 1986, Astrophys. Space Sci. 126, 243

Vereshchagin, S. V., Kraicheva, Z. T., Popova, E. I., Tutukov, A. V., and Yungel'son, L. R.: 1987, Sov. Astron. Lett. 13, 26

Vereshchagin, S. V., Kraicheva, Z. T., Popova, E. I., Tutukov, A. V., and Yungel'son, L. R.: 1988, Astrophys. Space Sci. 142, 245

Wasserman, I.: 1988, Astrophys. Space Sci. 142, 267

Worley, C. E. and Douglass, G.: 1984, The Washington Double Star Catalogue, U. S. Naval Observatory, Washington

Worley, C. E. and Heintz, W.: 1983, Fourth Catalogue of Orbits of Visual Binary Stars, U. S. Naval Observatory, Washington 